\title{
Repercussão Clínica da Reclassificação dos Carcinomas Diferenciados de Tireóide de Acordo com a 6a Edição do TNM
}

\section{RESUMO}

A classificação TNM segundo a UICC é usada para avaliar os resultados do acompanhamento do carcinoma de tireóide. A 6a edição modificou a descrição do tumor primário $(T)$, dos linfonodos regionais $(N)$ e dos grupos de estadiamento. O objetivo deste estudo foi comparar a habilidade das 5 a e 6 a edições em predizer resultados. As duas classificações foram aplicadas em uma análise retrospectiva de 90 pacientes do HUCFF. Sessenta e nove pacientes apresentavam carcinoma papilífero, 14 folicular, 4 células de Hürthle e 3 misto. Os pacientes foram acompanhados por um período médio de 58,3 meses. Ao final do acompanhamento, 49 pacientes estavam em remissão, 23 com doença persistente, 4 com recorrência tumoral, 11 com metástases e 3 evoluíram para o óbito. De acordo com a 6a edição, 19 pacientes foram classificados como T1, comparado com 7 pela 5a edição; 19 pacientes T2 comparado com 30; 14 classificados como T3 comparado com 10; 22 como T4 comparado com 27, e 16 pacientes como Tx. Ambas as edições mostraram remissões comparáveis para os estágios I, II e III. Para o estágio IV houve uma mudança significativa na remissão, entretanto não houve diferença comparando IV e IV C. (Arq Bras Endocrinol Metab 2007;51/5:825-831)

Descritores: Câncer de tireóide; Estadiamento; Prognóstico

\section{ABSTRACT}

Clinical Repercussion of the 6th $^{\text {th }}$ Edition TNM Staging System Classification on Differentiated Thyroid Carcinoma.

The TNM classification of UICC is used for predicting the outcome of thyroid cancer. The $6^{\text {th }}$ edition changed the description of primary tumor $(T)$, regional lymph node $(\mathrm{N})$ and the staging group. The aim of this study was to compare the ability of the $5^{\text {th }}$ and the $6^{\text {th }}$ editions to predict outcome. The two classifications were applied in a retrospective analysis of 90 patients from HUCFF. Sixtynine patients had papillary carcinoma, 14 follicular, 4 Hürthle cell, and 3 mixed. Patients were followed for a mean period of 58.3 months. At the end of followup, 49 patients were disease-free, 23 persisted with disease, 4 had cervical recurrence, 11 had metastases and 3 died. According to the $6^{\text {th }}$ edition, 19 patients were classified as T1, compared to 7 based on the $5^{\text {th }}$ edition; 19 patients were T2 compared to 30; 14 were T3 compared to 10; 22 were T4 compared to 27, and 16 patients were Tx. Both editions showed comparable remissions for stages I, II, and III. For the stage IV there was a significant change in remission, however there was no difference comparing IV and IV C. (Arq Bras Endocrinol Metab 2007;51/5:825-831)

Keywords: Thyroid cancer; Staging; Prognosis

$\mathrm{O}$ PACIENTES COM CARCINOMAS diferenciados da tireóide (papilífero e folicular) apresentam bom prognóstico após tratamento adequado. A sobrevida em 20 anos é de $95 \%$ para o carcinoma papilífero e de 70 a $80 \%$ para o folicular (1). No entanto, alguns pacientes apresentam alto risco de recorrência tumoral, ou mesmo de óbito, e o prognóstico é afetado por fatores relacionados ao paciente, ao tratamento e à própria doença. Dentre os fatores prognósticos destacam-se a idade ao diagnóstico, tipo histológico, tamanho tumoral, presença de invasão extra-tireoideana ou metástase a distância, administração de dose ablativa de iodo radioativo (131 I), manutenção de terapia supressiva do hormônio estimulador da tireóide (TSH) e artigo original

\author{
VANESSA M. VARANDAS \\ Sabrina Mendes Coelho \\ ana Paula V. Soeiro \\ Cláudia Medina Coell \\ Denise Pires de Carvalho \\ ALEXANDRU BUESCU \\ MÁRIO VAISMAN
}

Serviço de Endocrinologia (VMV, $S M C, A B \& M V)$ e Anatomia Patológica (APVS) do Hospital Universitário Clementino Fraga Filho, e Laboratório de Fisiologia Endócrina (DPC) do Instituto de Biofísica Carlos Chagas Filho, Universidade Federal do Rio de Janeiro; e Instituto de Medicina Social (CMC), Universidade Estadual do Rio de Janeiro, RJ.

Recebido em 08/08/06 Revisado em 09/01/07 Aceito em 23/01/07 
extensão da cirurgia $(2,3)$. A recidiva do câncer diferenciado da tireóide (CDT) ocorre em aproximadamente de 5 a $30 \%$ dos casos $(4,5)$. Em 5 a $20 \%$ dos casos ocorre localmente (leito tireoideano, linfonodos cervicais ou partes moles cervicais) e em 10 a $15 \%$ a distância (principalmente pulmão e osso).

Existem vários sistemas de estadiamento para o carcinoma tireoideano, visando estimar o prognóstico. Estes sistemas auxiliam na comparação de intervenções terapêuticas entre grupos semelhantes. $\mathrm{O}$ mais utilizado (tabela 1) é o desenvolvido pela International Union Against Cancer (UICC) e baseia-se na idade do paciente ao diagnóstico, no tipo histológico e no sistema TNM (6). Este sistema inclui 3 componentes: o tamanho do tumor primário $(\mathrm{T})$, a presença ou ausência de metástase para linfonodo regional $(\mathrm{N})$ e a presença ou ausência de metástase a distância $(\mathrm{M})$.

Em 2002, essa classificação foi reformulada de acordo com a padronização internacional de descrição e classificação dos estágios tumorais (7). O sistema TNM foi modificado especialmente quanto à descrição do tumor primário $(\mathrm{T})$ e a extensão para linfonodos regionais $(\mathrm{N})$. Desta forma, enquanto a $5 \underline{\text { a }}$ edição da UICC classifica tumor $\mathrm{Tl}$ como $\leq 1 \mathrm{~cm}$ de diâmetro e $\mathrm{T} 2>1$ $\mathrm{e} \leq 4 \mathrm{~cm}$, a 6 a edição define $\mathrm{Tl} \leq 2 \mathrm{~cm} \mathrm{e} \mathrm{T} 2>2 \mathrm{e} \leq 4$ cm. Pela 5a edição, T3 era classificado como tumor $>4$ $\mathrm{cm}$ sem extensão extra-tireoideana e T4, como todos os tumores com qualquer extensão extra-tireoideana. Por outro lado, a 6 a edição define T3 como tumor $>4 \mathrm{~cm}$ em seu maior diâmetro, ou qualquer tumor com mínima extensão extra-tireoideana (isto é, invasão de tecido conjuntivo peri-tireoideano e/ou músculo esternotireoideano); e T4 como qualquer tumor com extensa invasão extra-tireoideana. T4 é subdividido em T4a, quando há invasão de tecidos moles subcutâneos, laringe, traquéia, esôfago e/ou nervo laríngeo recorrente; e T4b, quando há acometimento da fáscia prévertebral, vasos mediastínicos e/ou artéria carótida.

Quanto às modificações do acometimento de linfonodos $(\mathrm{N})$, a $5 \underline{a}$ edição classifica Nla como metástase cervical unilateral e Nlb como metástases bilaterais, localizadas na linha média ou cervical contra-lateral ou linfonodos mediastínicos. Entretanto, a 6aㅗ edição define como Nla apenas metástases para linfonodos ipsilaterais pré-traqueais, para-traqueais e prélaringeos e Nlb como os demais casos. Essas modificações quanto ao $\mathrm{T}$ e $\mathrm{N}$ determinaram alteração nos grupos de estadiamento (tabela 1 ).

O objetivo deste trabalho é avaliar, através de análise retrospectiva, a capacidade da nova classificação

Tabela 1. Classificação do carcinoma da tireóide pela $5^{\mathrm{a}}$ e $6^{\mathrm{a}}$ edições da UICC.

\begin{tabular}{|c|c|c|}
\hline & $5^{a}$ edição & $6^{a}$ edição \\
\hline Tx & Sem definição do tamanho tumoral & Sem definição do tamanho tumoral \\
\hline $\mathrm{T} 1$ & $<1 \mathrm{~cm}$, restrito à tireóide & $<2 \mathrm{~cm}$, restrito à tireóide \\
\hline T2 & $>1 \mathrm{e} \leq 4 \mathrm{~cm}$, restrito à tireóide & $>2 \mathrm{e} \leq 4 \mathrm{~cm}$, restrito à tireóide \\
\hline T3 & $>4 \mathrm{~cm}$, restrito à tireóide & $\begin{array}{l}>4 \mathrm{~cm} \text {, restrito à tireóide ou mínima } \\
\text { extensão extra-tireoideana }\end{array}$ \\
\hline T4 & $\begin{array}{l}\text { Qualquer tamanho, com extensão } \\
\text { extra-tireoideana }\end{array}$ & $\begin{array}{l}\text { T4a: tumor com extensão além da cápsula, } \\
\text { invasão de tecido mole subcutâneo, } \\
\text { laringe, traquéia, esôfago ou nervo } \\
\text { laringeo recorrente } \\
\text { T4b: tumor invadindo fáscia pré-vertebral, } \\
\text { vasos mediastínicos ou artéria carótida }\end{array}$ \\
\hline $\mathrm{Nx}$ & $\begin{array}{l}\text { Sem definição de acometimento de } \\
\text { linfonodos }\end{array}$ & $\begin{array}{l}\text { Sem definição de acometimento de } \\
\text { linfonodos }\end{array}$ \\
\hline N1 & Metástase para linfonodo regional & Metástase para linfonodo regional \\
\hline $\mathrm{N} 1 \mathrm{a}$ & $\begin{array}{l}\text { Metástase para linfonodo cervical, } \\
\text { unilateral }\end{array}$ & $\begin{array}{l}\text { Metástase nível VI (pré e para-traqueal, } \\
\text { pré-laríngeo e de Delphian) }\end{array}$ \\
\hline N1b & $\begin{array}{l}\text { Metástases bilaterais, localizadas na } \\
\text { linha média, cervical contra-lateral ou } \\
\text { mediastínica }\end{array}$ & $\begin{array}{l}\text { Metástases em outros cervicais unilaterais, } \\
\text { bilaterais ou contra-laterais ou mediastí- } \\
\text { nicas }\end{array}$ \\
\hline$M x$ & $\begin{array}{l}\text { Sem definição de metástases à dis- } \\
\text { tância }\end{array}$ & Sem definição de metástases à distância \\
\hline M0 & Sem metástases à distância & Sem metástases à distância \\
\hline M1 & Metástases à distância & Metástases à distância \\
\hline Estágio I & $\mathrm{M} 0<45$ anos; $\mathrm{T} 1 \geq 45$ anos & $\mathrm{TxNxM0}<45$ anos; T1N0M0 $\geq 45$ anos \\
\hline Estágio II & $\mathrm{M} 1<45$ anos; T2 ou T3 $\geq 45$ anos & TxNxM1 $<45$ anos; T2N0M0 $\geq 45$ anos \\
\hline Estágio III & T4 ou $N 1 \geq 45$ anos & T3N0M0 > 45 anos; T1-3N1aM0 $\geq 45$ anos \\
\hline Estágio IV & M1 $\geq 45$ anos & $\begin{array}{l}\text { IVA: T1-3N } 1 b M 0>45 \text { anos; T4aN0-1 M0 } \geq \\
45 \text { anos } \\
\text { IVB: T4bNxM0 } \geq 45 \text { anos } \\
\text { IVC: } T x N x M 1 \geq 45 \text { anos }\end{array}$ \\
\hline
\end{tabular}


em predizer o prognóstico nos diferentes estágios dos diferentes grupos de estadiamento do carcinoma diferenciado de tireóide (CDT), comparando-a com a da $5^{\mathrm{a}}$ edição.

\section{PACIENTES E MÉTODOS}

\section{População do estudo}

Foi realizado levantamento de todos os pacientes com CDT submetidos a tratamento complementar com radioiodo de 1994 a 2004 no Serviço de Medicina Nuclear do Hospital Universitário Clementino Fraga (HUCFF). Foram excluídos os pacientes que não permaneceram em acompanhamento neste hospital. A classificação tumoral foi determinada de acordo com o resultado histopatológico (pTNM).

Foram incluídos neste estudo noventa pacientes (71 mulheres e 19 homens), com idade ao diagnóstico variando de 13 a 78 anos (mediana de $44 \mathrm{DP} \pm$ 16,76). Quanto ao tipo histológico, 69 foram classificados como papilíferos, 14 como foliculares, 4 como células de Hürthle e 3 papilífero associado a folicular. Trinta e quatro pacientes foram submetidos a tireoidectomia subtotal, 37 a tireoidectomia total, 18 realizaram tireoidectomia total simultaneamente com esvaziamento ganglionar e somente um paciente realizou tireoidectomia subtotal com esvaziamento ganglionar. Dos pacientes submetidos à cirurgia mais conservadora no primeiro momento, 12 realizaram tireoidectomia total e 6 realizaram também o esvaziamento ganglionar. Dentre os 37 pacientes submetidos a tireoidectomia total no primeiro tempo, 2 realizaram esvaziamento ganglionar posteriormente.

Os pacientes foram acompanhados por um período médio de 58,28 meses (variando de 6 a 288 meses), de acordo com protocolo estabelecido pelo Serviço de Endocrinologia do HUCFF. Após 6 a 12 meses do tratamento inicial (cirurgia e radioiodoterapia), os pacientes foram submetidos a pesquisa de corpo inteiro (PCI) e dosagem de tireoglobulina $(\mathrm{Tg})$ sérica em hipotireoidismo. Os pacientes com PCI sem captação anormal do radiotraçador e Tg sérica estimulada inferior a $2 \mathrm{ng} / \mathrm{ml}$ foram considerados em remissão e acompanhados com dosagem seriada de $\mathrm{Tg}$ em vigência de terapia supressora de TSH, e, nos últimos anos, com USG cervical anual. No caso de Tg elevada (em supressão $>1 \mathrm{ng} / \mathrm{ml}$ ou em hipotireoidismo $>2$ $\mathrm{ng} / \mathrm{ml}$ ) e/ou PCI com captação anormal, foram realizadas investigação e terapia específicas. A dosagem da $\mathrm{Tg}$ foi realizada por quimioluminescência, com sensibilidade analítica de $0,2 \mathrm{ng} / \mathrm{ml}$, funcional de 0,9 $\mathrm{ng} / \mathrm{ml}$, variação inter-ensaio de até $8,8 \%$ e intra-ensaio inferior a $6 \%$ para valores superiores a $2 \mathrm{ng} / \mathrm{ml}$. O TSH foi dosado por ensaio de $3^{a}$ geração, por quimioluminescência, com faixa de normalidade entre $0,4 \mathrm{e}$ $4,0 \mu \mathrm{UI} / \mathrm{ml}$. O anticorpo anti-Tg foi pesquisado em todas as amostras coletadas para dosagem de $\mathrm{Tg}$. O método de detecção utilizado foi de quimioluminescência, com sensibilidade analítica de $10 \mathrm{IU} / \mathrm{ml}$, variação inter-ensaio de até 9,1\% e intra-ensaio de $3,9 \%$.

\section{Análise de dados}

\section{Avaliação de concordância}

A concordância entre as duas edições da classificação foi aferida através da estatística kappa ponderado, empregando-se os pesos derivados da seguinte fórmula: $1-|\mathrm{i}-\mathrm{j}| / 3$; onde $\mathrm{i}=$ estádio pela $5^{\mathbf{a}}$ edição da classificação e $j=$ estádio pela $\sigma^{a}$ edição. A estatística kappa permite avaliar a extensão da concordância entre dois examinadores (nesse estudo as duas edições da classificação), que excede a que seria esperada somente devido ao acaso (12).

Os intervalos de 95\% de confiança (IC 95\%) do kappa foram calculados empregando-se a rotina kapci (7), desenvolvida para o software Stata (17). Para a interpretação do kappa foi empregada a classificação proposta por Shrout (14), que considera as seguintes classes de concordância: $\kappa<0,1$ concordância virtualmente ausente; $\kappa=0,10$ a 0,40 concordância fraca; $\kappa=0,41$ a 0,60 concordância discreta; $\kappa=0,61$ a 0,80 concordância moderada; $\kappa=0,81$ a 1,0 concordância substancial.

\section{Avaliação de prognóstico}

A incidência de persistência após o tratamento inicial foi calculada globalmente e segundo estádio em cada edição da classificação, sendo a diferença entre estádios avaliadas através do teste de Fisher. Adicionalmente, foram avaliadas as curvas de sobrevida de Kaplan-Meier (1) considerando como desfecho o tempo (em anos) até a ocorrência de recidiva, óbito ou censura (pacientes perdidos de observação ou que não apresentaram os eventos de interesse até o final do período de observação). As curvas de sobrevida segundo estádio tumoral em cada edição da classificação foram comparadas empregando-se o teste do log-rank. Por fim, considerando que o maior número de pacientes se concentrou no grupo de estádio tumoral I, optamos por comparar a curva desse grupo com a dos demais grupos analisados em conjunto. Adicionalmente, foram calculadas hazard ratios por meio do ajuste de modelos de Cox (Cox proportional hazards model) empregando 
como referência o grupo de estádio tumoral I. Para as análises de sobrevida apenas foram considerados os 71 pacientes que não apresentaram persistência do tumor após o tratamento inicial. Os dados foram analisados empregando o programa Stata (versão 8.0) (14). Para a avaliação da significância estatística empregou-se o teste bi-caudal sendo considerado estatisticamente significativo um valor de $\mathrm{p}(\alpha) \leq 0,05$.

\section{RESULTADOS}

\section{Análise de concordância}

Dentre os 90 pacientes incluídos no estudo, 16 não apresentavam definição do tamanho tumoral ao exame histopatológico, sendo então classificados como Tx. Dos 74 pacientes restantes, 7 foram classificados de acordo com a $5^{\text {a }}$ edição do TNM como Tl, 30 como T2, 10 como T3 e 27 como T4. Pela $6^{\text {a }}$ edição, 19 foram definidos com T1, 19 como T2, 14 como T3 e 22 como T4 (tabela 2 ). Dezoito pacientes $(24,32 \%)$ apresentaram mudança da classificação quanto ao tamanho tumoral. Dos 19 pacientes reclassificados como T1 pela $6^{\mathrm{a}}$ edição, 12 eram anteriormente T2; dentre os 19 reclassificados como T2, somente 1 era classificado como T3. Nos 14 pacientes do grupo T3, 5 eram definidos inicialmente como T4 (figura 1). Por fim, 22 pacientes permaneceram como T4, sendo 20 pacientes classificados como T4a e 2 como T4b.

Em relação ao acometimento de linfonodos, 56 foram definidos como N0 em ambas as edições. Pela $5^{\text {a }}$ edição, 30 foram classificados como Nla e 4 como Nlb. Somente em um caso houve mudança da classificação: um paciente inicialmente classificado com $\mathrm{Nla}$ foi reclassificado como Nlb pela $6^{\mathrm{a}}$ edição.

De acordo com a $5^{\text {a }}$ edição de estadiamento tumoral, 44 pacientes foram classificados como estágio I, 25 como II, 19 como III e 2 como IV. Pela última edição, 49 foram reclassificados como I, 9 como II, 16 como III e 16 como IV, sendo 14 IV A e 2 IV C (tabela 3 ). Trinta pacientes $(33,3 \%)$ tiveram mudança de estágio: dos pacientes classificados inicialmente com estágio II, 5 foram reclassificados como I e 11 como estágio III; e 14 pacientes classificados como estágio III foram reclassificados como IV A. Considerando-se os quatro níveis do estadiamento tumoral, i.e., os níveis IV A e IV C da $6^{\text {a }}$ edição foram tratados conjuntamente, observou-se um kappa ponderado de 0,70 (IC 95\%: 0,69-0,72), indicando concordância moderada.

\section{Avaliação do prognóstico}

Ao término do estudo, 49 pacientes $(54,44 \%)$ apresentaram critério de remissão, $23(25,55 \%)$ de persistência tumoral, $4(4,44 \%)$ evoluíram com recidiva cervical, $11(12,22 \%)$ com metástases a distância e 3 (3,33\%) evoluíram para óbito.

Não foi observada nenhuma associação significativa entre o estádio e a ocorrência de persistência independentemente da edição da classificação empregada (tabela 4).

Na figura 1 são apresentadas as curvas de sobrevida de acordo com o estádio tumoral pela 5a edição (a) e 6a edição (b). Em ambos os casos, observa-se uma diferença significativa entre as curvas, especialmente no que se refere ao estádio tumoral IV da $5 \underline{a}$ edição e o estádio IV C da 6a em relação às demais curvas. A transferência de casos do estádio II na $5 \underline{\text { a }}$ edição para o I da 6a não modificou o comportamento desse grupo em termos de sobrevida. Já o grupo de estádio III na sexta edição aparentemente apresentou comportamento próximo ao do estádio II da quinta edição, o que era esperado já que esse grupo ganhou pacientes anteriormente classificados no grupo II e teve pacientes deslocados para o grupo IV A. O grupo de estádio IV A na sexta edição apresentou comportamento que se assemelha ao grupo III da quinta edição. O comportamento da curva dos pacientes classificados no estádio II na sexta edição ficou muito errático em função do número reduzido de casos classificados nesse grupo, prejudicando a interpretação da mesma.

Todos os pacientes classificados no grupo de estádio tumoral I sobreviveram ao primeiro ano de seguimento, independentemente da edição empregada. Quando esse grupo é comparado com os demais, verifica-se que a nova edição apresenta um poder de

Tabela 2. Classificação da extensão tumoral (T) de acordo com a $5^{a}$ e a $6^{a}$ edições da UICC.

\begin{tabular}{|c|c|c|c|c|c|}
\hline & T1 (5ª edição) & $\begin{array}{c}\text { T2 } \\
\text { (5ª edição) }\end{array}$ & $\begin{array}{c}\text { T3 } \\
\text { (5 edição) }\end{array}$ & $\begin{array}{c}\text { T4 } \\
\text { (5 edição) }\end{array}$ & Total pela $6^{a}$ edição \\
\hline T1 (6 ${ }^{a}$ edição) & 7 & 12 & - & - & 19 \\
\hline T2 (6 $6^{\mathrm{a}}$ edição) & - & 18 & 1 & & 19 \\
\hline T3 (6 $6^{a}$ edição) & - & - & 9 & 5 & 14 \\
\hline T4 (6 ${ }^{\mathrm{a}}$ edição) & - & - & - & 22 & 22 \\
\hline Total pela $5^{\mathrm{a}}$ edição & 7 & 30 & 10 & 27 & 74 \\
\hline
\end{tabular}




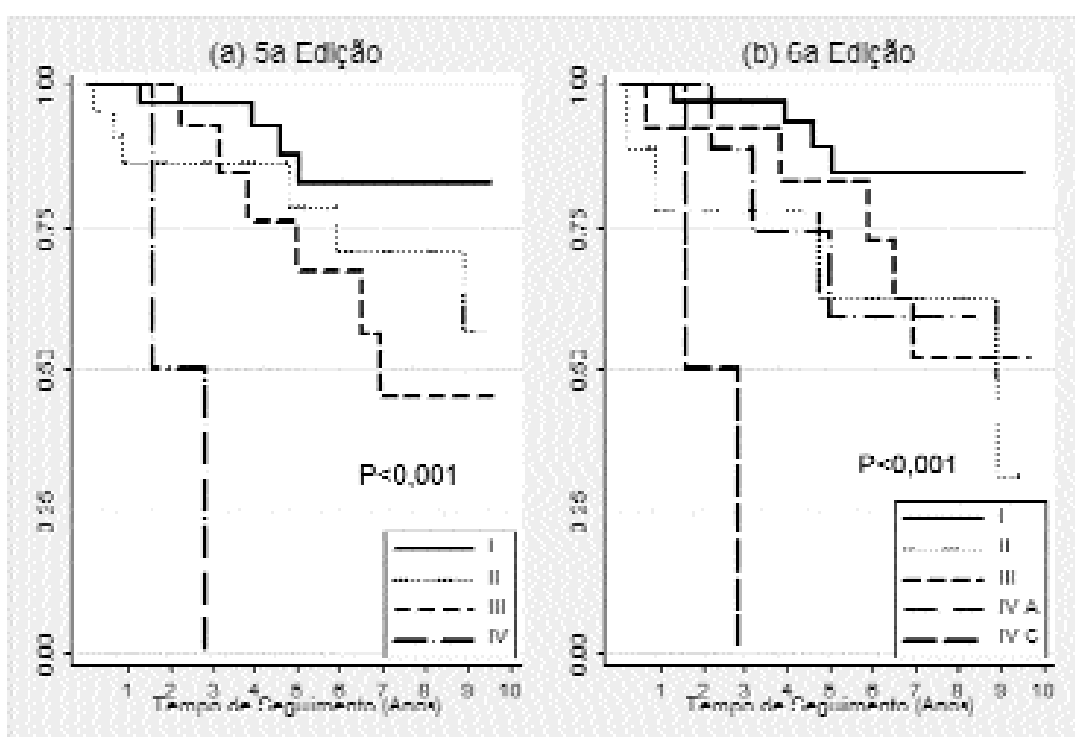

Figura 1. Curvas de Sobrevida de Kaplan-Meier segundo estádio tumoral pela $5^{a}$ (a) e $6^{a}$ (b) edições da classificação TMN.

Tabela 3. Estadiamento do CDT de acordo com a $5^{\mathrm{a}}$ e a $6^{\mathrm{a}}$ edições da UICC.

\begin{tabular}{|c|c|c|c|c|c|}
\hline & $\mathbf{I}$ & II & III & IV & Total pela $5^{a}$ edição \\
\hline I & 44 & 0 & 0 & 0 & 44 \\
\hline II & 5 & 9 & 11 & 0 & 25 \\
\hline III & 0 & 0 & 5 & 14 & 19 \\
\hline IV & 0 & 0 & 0 & 2 & 2 \\
\hline Total pela $6^{a}$ edição & 49 & 9 & 16 & 16 & 90 \\
\hline
\end{tabular}

Tabela 4. Incidência de persistência segundo estádio tumoral pelas duas edições.

\begin{tabular}{lcc}
\hline Estadio & $\begin{array}{c}\text { Incidência de Persistência } \\
\text { Total de Pacientes }\end{array}$ & N (\%) \\
$\mathbf{5}^{\mathbf{a}}$ Edição & 44 & $12(27,3 \%)$ \\
I & 25 & $3(12,0 \%)$ \\
II & 19 & $4(21,0 \%)$ \\
III & 2 & - \\
IV & & 0,48 \\
P-Valor & 49 & $12(24,5 \%)$ \\
6 Edição & 9 & - \\
I & 16 & $3(18,8 \%)$ \\
II & 14 & $4(28,6 \%)$ \\
III & 2 & - \\
IV A & & 0,47 \\
IV C & & \\
P-Valor & & \\
\hline
\end{tabular}

discriminação ligeiramente melhor que o da a quinta edição (figura 2). Esses resultados são corroborados quando se analisam os valores das hazard ratios (HR), sendo observado um risco 3,5 (IC 95\% 1,3-9,6) maior de evoluir para recidiva ou óbito no grupo de pacientes classificados nos grupos de estádio tumoral de II a IV C em relação ao grupo com estádio I, quando se emprega a sexta edição da classificação. Já para a quinta edição observa-se uma HR igual a 2,9 (IC 95\% $1,0-8,0)$.
DISCUSSĀO

Estudos de fatores prognósticos do carcinoma diferenciado de tireóide apresentam, por vezes, resultados conflitantes. Entretanto, a idade ao diagnóstico é o fator independente mais importante na maioria dos estudos $(8,9)$. Em geral, pacientes mais velhos tendem a apresentar tumores localmente mais agressivos, maior incidência de metástase a distância já ao diagnóstico, variantes histológicas mais agressivas e menor captação de iodo pelo tumor (14).

Entre todos os sistemas de estadiamento, o TNM é o mais utilizado por ser prático e de fácil aplicação (10). Entretanto apresenta algumas limitações, dentre elas não considerar os subtipos histológicos e tipo de intervenção terapêutica.

Uma das principais modificações da nova classificação da UICC foi quanto à definição de $\mathrm{Tl}$, ampliando o tamanho tumoral de 1 para até $2 \mathrm{~cm}$ de diâmetro. Como conseqüência disso, foi observado um aumento significativo do número de pacientes reclassificados como Tl na nossa casuística, uma vez que 12 pacientes inicialmente classificados como $\mathrm{T} 2$ foram redefinidos como Tl pela $6^{a}$ edição. Uma outra modificação observada nesta nova edição foi a inclusão de tumores com 


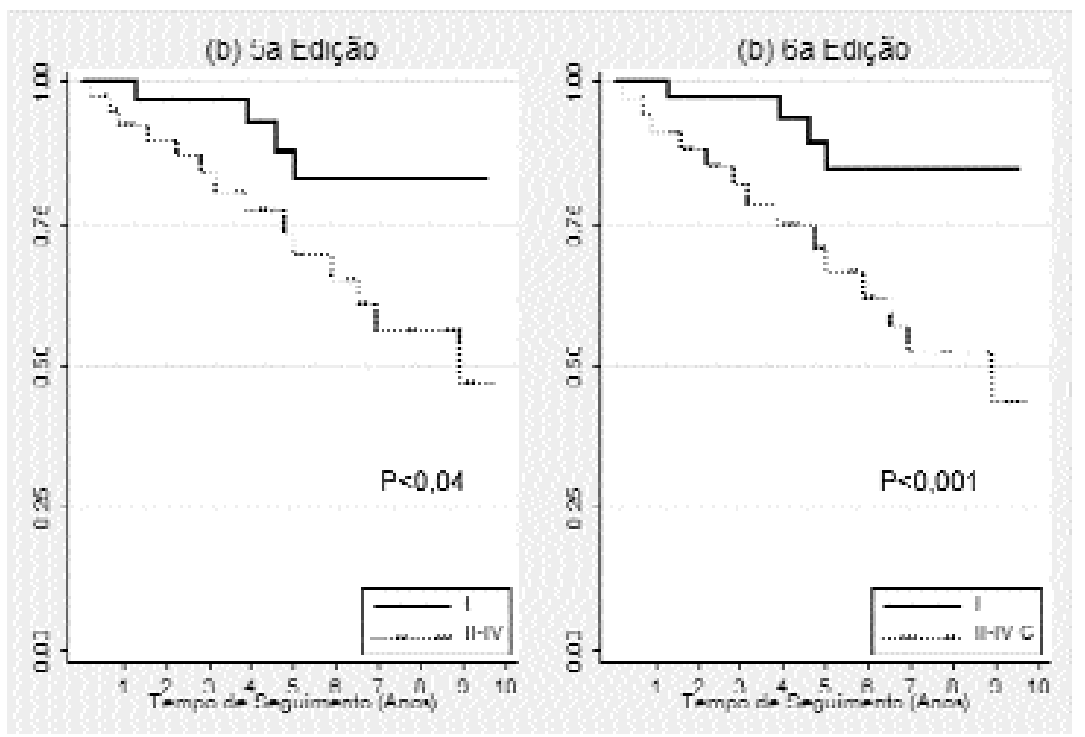

Figura 2. Curvas de Sobrevida de Kaplan-Meier segundo estádio tumoral pela $5^{a}$ (a) (I vs. II-IV) e $6^{a}$ (b) (I vs. II-IV C) edições da classificação TMN.

mínima extensão extra-tireoideana no grupo T3. Com esta mudança, do total de 27 pacientes inicialmente classificados como T4, 5 foram redefinidos como T3.

Em relação ao acometimento de linfonodos, a nova edição limitou o grupo Nla para linfonodos restritos às cadeias pré e para-traqueais unilaterais. Não houve repercussão significativa desta mudança no grupo de pacientes avaliados, com apenas 1 único caso de modificação de Nla para Nlb.

As modificações do estadiamento da $6^{a}$ edição restringem-se aos pacientes com idade ao diagnóstico $\geq 45$ anos. Neste grupo, os pacientes com tumor T3 foram re-classificados do estágio II para o III; e com tumor T4, do estágio III para IV. Os pacientes com metástases para linfonodos, porém sem metástases a distância, eram sempre considerados pela $5^{\text {a }}$ edição como estágio III. Entretanto, pela $6^{a}$ edição, aqueles classificados como Nlb foram redefinidos como estágio IV. Dos 90 pacientes estudados, 6 apresentavam tumor T3 e foram reclassificados do estágio II para o III e 14 apresentavam tumor T4 e foram reclassificados do III para o IV. Destes 14, l paciente também apresentou modificação quanto à redefinição de $\mathrm{N}$.

Além das modificações já descritas, mudanças no estadiamento dos pacientes, como idade ao diagnóstico $\geq 45$ anos, também ocorrem em decorrência da alteração da definição do tamanho tumoral. Desta forma, 4 pacientes inicialmente classificados como T2N0M0 (estágio II), foram redefinidos como T1N0M0 (estágio I). Dois pacientes definidos pela $5^{\text {a }}$ edição como T2NlaM0 (estágio I) foram então reclassificados como TlNlaM0 (estágio II).
Ao analisar a taxa de remissão, não foi observada modificação significativa para os estágios I, II e III. No entanto, o estágio IV apresentou um aumento da taxa de remissão pela $6^{\text {a }}$ edição (de 0 para $43,75 \%$ ). Isto pode ser explicado pelo fato de a $5^{\text {a }}$ edição restringir o estágio IV a pacientes com idade ao diagnóstico $\geq 45$ anos e metástases a distância. Já a $6^{a}$ edição inclui, também, pacientes sem metástases a distância, porém com tumor T4 e/ou acometimento de linfonodo Nlb.

No presente estudo, foi observada uma baixa taxa de remissão para os estágios I,II e III, por ambas as edições, quando comparado com a literatura (11). Este achado pode ser, pelo menos em parte, explicado pelo tratamento inicial mais conservador, associado a um risco elevado de recidiva (12). Aproximadamente $40 \%$ dos pacientes foram submetidos a cirurgias mais conservadoras. Destes, $50 \%$ foram re-operados, porém o tempo entre as cirurgias foi maior que 6 meses em uma significativa proporção. Além disso, o intervalo entre a intervenção cirúrgica e a radioiodoterapia também pode ter contribuído. A taxa de remissão dos pacientes dos estágios III e IV, que foram submetidos inicialmente a tireoidectomia total no primeiro momento, foi de 47,83\%. Já no grupo de pacientes em que foi realizada cirurgia mais conservadora no primeiro momento, a taxa de remissão foi de apenas 25\%. Nos estágios I e II não foram observados aparentes benefícios da totalização. A taxa de remissão do subgrupo de pacientes tratados de forma conservadora foi de $78,57 \%$ e nos pacientes re-operados foi de $53,84 \%$. Isto pode ser justificado pela indicação da 
segunda cirurgia nos pacientes com forte suspeita de recidiva ou persistência tumoral.

Döbert e cols. (13) avaliaram retrospectivamente 169 pacientes com CDT. Foi constatado que a taxa de pacientes livres de doença foi discretamente menor no grupo re-classificado como Tl pela $6^{\mathrm{a}}$ edição, no entanto não foram observadas diferenças nos grupos T2 e T3. Neste trabalho, não houve paciente classificado como T4. Entretanto, em nosso estudo, não foi observada diminuição significativa da taxa de remissão para nenhum dos grupos, exceto para o grupo T4.

Desta forma, observamos que as duas classificações apresentaram uma concordância apenas moderada, e que o remanejamento de alguns pacientes para o grupo I pareceu ter sido adequado, melhorando a capacidade de discriminar pacientes. No entanto, acreditamos que o tamanho da amostra possa ter limitado a avaliação das demais mudanças.

\section{REFERÊNCIAS}

1. Bland JM, Altman DG. Survival probabilities (the KaplanMeier method). BMJ 1998;317:1572-80.

2. Brierley JD, Panzarella T, Tsang RW, Gospodarowicz MK, O'Sullivan B. A comparison of different staging systems predictability of patient outcome. Thyroid carcinoma as an example. Cancer 1997;15:2414-23.

3. Cohen J. A coefficient of agreement for nominal scales. Educ Psychol Measurements 1960;20:37-46.

4. Denoix PF. Tumor, node, and metastasis (TNM). Bull Inst Nat Hyg 1944;1:1-69.

5. Döbert N, Menzel C, Oeschger S, Grünwald F. Differentiated thyroid carcinoma: the new UICC $6^{\text {th }}$ edition TNM classification system in a retrospective analysis of 169 patients. Thyroid 2004; $14: 65-70$.

6. Emerick GT, Duh QY, Siperstein $A E$, Burrow GN, Clark OH. Diagnosis, treatment and outcome of follicular thyroid carcinoma. Cancer 1993;72:3287-95.
7. Gilliland FD, Hunt WC, Morris DM, Key CR. Prognostic factors for thyroid carcinoma: a population-based study of 15,698 cases from the Surveillance, Epidemiology and End Results (SEER) program 1973-1991. Cancer 1997;79:564-73.

8. Hamming JF, Van de Velde CJ, Goslings BM, Shelfhout LJ, Fleuren GJ, Hermans J. Prognosis and morbidity after total thyroidectomy for papillary and follicular and medullary thyroid cancer. Eur J Cancer Clin Oncol 1989;25:1317-23.

9. Loh KC, Greenspan FS, Gee L, Miller TR, Yeo PPB. Pathological tumor-node-metastasis (pTNM) staging for papillary and follicular thyroid carcinomas: A retrospective analysis of 700 patients. J Clin Endocrinol Metab 1997;82:3553-62.

10. Mazzaferri EL. An overview of the management of papillary and follicular thyroid carcinoma. Thyroid 1999;9:421-7.

11. Mazzaferri EL, Jhiang SM. Long-term impact of initial surgical and medical therapy on papillary and follicular thyroid cancer. Am J Med 1994;97:418-28.

12. Reichenheim ME. Confidence intervals for the kappa statistic. Stata J 2004;4:421-8.

13. Schlumberger M. Papillary and follicular thyroid carcinoma. N Engl J Med 1998;338:297-306

14. Schlumberger M, Pacini F. Thyroid tumors. Paris: Éditions Nucléon, 1999. p. 317

15. Shrout PE. Measurement reliability and agreement in psychiatry. Stat Methods Med Res 1998;7:301-17.

16. Simpson WJ, McKinney SE, Carruthers JS, Gospodarowicz MK, Sutcliffe SB, Panzarella T. Papillary and follicular thyroid cancer - prognostic factors in 1,578 patients. Am J Med 1987; 83:479-88.

17. Sobin $\mathrm{LH}$, Wittekind $\mathrm{CH}$. TNM classification of malignant tumors. $6^{\text {th }}$ edition. New York: Wiley-Liss, 2002. pp. 52-6.

18. StataCorp. Stata Statistical Software: Release 8.0. College Station, TX: Stata Corporation, 2003.

19. Yildirim E. A model for predicting outcomes in patients with differentiated thyroid cancer and model performance in comparison with other classification systems. J Am Coll Surg 2005;200:378-92.

Endereço para correspondência:

Mário Vaisman

Rua Venâncio Flores 368, apto. 302

22441-090 Rio de Janeiro, RJ

Fax: (21) 2512-6474

E-mail: vaisman@hucff.ufrj.com.br 